\title{
Using an artificial neural network to predict traumatic brain injury
}

\author{
*Andrew T. Hale, MS, ${ }^{1,2}$ David P. Stonko, MS, MD, ${ }^{2,3}$ Jaims Lim, MD, ${ }^{2}$ \\ Oscar D. Guillamondegui, MD, MPH, ${ }^{2,3,6}$ Chevis N. Shannon, $\mathrm{PhD},{ }^{2,6,7}$ and \\ Mayur B. Patel, MD, MPH2,4-6
}

\begin{abstract}
${ }^{1}$ Vanderbilt University School of Medicine, Medical Scientist Training Program; ${ }^{2}$ Vanderbilt University School of Medicine, Nashville, Tennessee; ${ }^{3} \mathrm{~J} o h n s$ Hopkins Medical Institute, Johns Hopkins University School of Medicine, Baltimore, Maryland; ${ }^{4}$ Division of Trauma, Emergency General Surgery, and Surgical Critical Care, Departments of Surgery and Hearing and Speech Sciences, Section of Surgical Sciences, Vanderbilt University Medical Center; ${ }^{5}$ Center for Health Services Research, Vanderbilt Brain Institute, Vanderbilt University Medical Center and Geriatric Research, Education and Clinical Center Service, Surgical Service, Department of Veterans Affairs Medical Center, Tennessee Valley Health Care System; ${ }^{6}$ Department of Neurosurgery, Vanderbilt University Medical Center and Division of Pediatric Neurosurgery, Monroe Carell Jr. Children's Hospital of Vanderbilt University; and 'Surgical Outcomes Center for Kids, Monroe Carell Jr. Children's Hospital of Vanderbilt University, Nashville, Tennessee
\end{abstract}

OBJECTIVE Pediatric traumatic brain injury (TBI) is common, but not all injuries require hospitalization. A computational tool for ruling in patients who will have a clinically relevant TBI (CRTBI) would be valuable, providing an evidencebased way to safely discharge children who are at low risk for a CRTBI. The authors hypothesized that an artificial neural network (ANN) trained on clinical and radiologist-interpreted imaging metrics could provide a tool for identifying patients likely to suffer from a CRTBI.

METHODS The authors used the prospectively collected, publicly available, multicenter Pediatric Emergency Care Applied Research Network (PECARN) TBI data set. All patients under the age of 18 years with TBI and admission head CT imaging data were included. The authors constructed an ANN using clinical and radiologist-interpreted imaging metrics in order to predict a CRTBI, as previously defined by PECARN: 1) neurosurgical procedure, 2) intubation > 24 hours as direct result of the head trauma, 3) hospitalization $\geq 48$ hours and evidence of TBI on a CT scan, or 4) death due to TBI.

RESULTS Among 12,902 patients included in this study, 480 were diagnosed with CRTBI. The authors' ANN had a sensitivity of $99.73 \%$ with precision of $98.19 \%$, accuracy of $97.98 \%$, negative predictive value of $91.23 \%$, false-negative rate of $0.0027 \%$, and specificity for CRTBI of $60.47 \%$. The area under the receiver operating characteristic curve was 0.9907 . CONCLUSIONS The authors are the first to utilize artificial intelligence to predict a CRTBI in a clinically meaningful manner, using radiologist-interpreted CT information, in order to identify pediatric patients likely to suffer from a CRTBI. This proof-of-concept study lays the groundwork for future studies incorporating iterations of this algorithm directly into the electronic medical record for real-time, data-driven predictive assistance to physicians.

https://thejns.org/doi/abs/10.3171/2018.8.PEDS18370

KEYWORDS TBI; pediatrics; machine learning; artificial intelligence; trauma

$\mathrm{T}$ RAUMATIC brain injury (TBI) affects thousands of children in the United States every year. ${ }^{33}$ Despite the large number of children who experience TBI, only a small percentage actually require hospitalization or prolonged surveillance. ${ }^{30}$ However, identifying which patients do require monitoring compared with those who can be safely discharged from the emergency department remains an important unanswered question. Thus, creation of a tool for identifying patients at risk for clinically relevant TBI (CRTBI) could provide an evidence-based

ABBREVIATIONS ANN = artificial neural network; $\mathrm{AUC}=$ area under the curve; $\mathrm{CRTBI}=$ clinically relevant $\mathrm{TBI} ; \mathrm{EMR}=$ electronic medical record; $\mathrm{NPV}=$ negative predictive value; $P E C A R N=$ Pediatric Emergency Care Applied Research Network; $R O C=$ receiver operator characteristic; $T B I=$ traumatic brain injury.

SUBMITTED June 13, 2018. ACCEPTED August 8, 2018

INCLUDE WHEN CITING Published online November 2, 2018; DOI: 10.3171/2018.8.PEDS18370.

${ }^{*}$ A.T.H. and D.P.S. contributed equally to this work. 
mechanism for early safe discharge and potentially reduce unnecessary healthcare expenditures.

The Pediatric Emergency Care Applied Research Network (PECARN) $)^{19}$ is a consortium of 25 hospitals that developed a decision-making score based on head CT findings. ${ }^{15}$ Numerous studies have independently published on these data in an effort to develop predictive metrics to guide treatment of children with TBI; however, none have used artificial neural networks (ANNs). $3,4,7,10,14-16,20,21$, $25,27,28$ ANNs are a type of machine-learning algorithm that have been widely used in clinical medicine.,5,69 They are often more useful than conventional statistical methods because: 1) ANNs can take any number of input variables and predict any number of outcomes; 2) they are capable of improving their predictive ability over time as they are exposed to new data; 3 ) they benefit from internal validation and testing; and 4) they tend to have stronger discriminant ability compared to conventional statistics. ${ }^{1,13,31,40}$

Leveraging this technology, we created a model that combines clinical and radiologist-interpreted CT data to predict whether or not a pediatric patient will experience a CRTBI. We quantify the accuracy and error of this algorithm and provide an open-source software package to enable prediction generation and validation. We expand on previous PECARN predictive studies by utilizing a combination of demographic, clinical, and radiologistinterpreted CT data to investigate CRTBIs in pediatric patients using an ANN. For the present study we hypothesized that we could train an ANN on clinical and radiographic data to identify which pediatric TBI patients with head CT scans are at risk for a CRTBI.

\section{Methods \\ Study Population}

This study utilized the prospective PECARN study of children with CRTBI, as described previously. ${ }^{15,19}$ The PECARN TBI study enrolled patients under the age of 18 who experienced nonpenetrating (i.e., blunt) head trauma who presented to the emergency department between 2004 and 2006 and had admission head CT imaging classification. All data analyzed in this study were de-identified, and our study was approved by the Vanderbilt University Institutional Review Board. We included patients who had complete data available for all variables of interest and thus did not impute any missing variables. Head CT scanning was performed in 14,969 patients, 12,902 of whom had complete imaging information.

\section{Analysis and Included Variables}

Descriptive statistics including Pearson correlation and t-test were used to evaluate the normally distributed cohort variables. Statistical significance was set a priori at $\mathrm{p}$ $<0.05$. The input variables included in our ANN were as follows: 1) mechanism of injury (e.g., motor vehicle collision, pedestrian struck by a moving vehicle, bicycle rider struck by an automobile, bicycle collision or fall from bicycle, other wheeled transport crash, fall to ground from standing/walking/running, walked or ran into stationary object, fall from an elevation, fall down stairs, sports, assault, object struck head [accidental], and other etiology of injury); 2) severity of injury mechanism (low [e.g., fall from ground level and walked/ran into a stationary object], moderate [any other mechanism], high [e.g., motor vehicle collision with patient ejection, death of another passenger, or rollover; pediatric or bicyclist without helmet struck by motor vehicle; and fall $>5$ feet for patients 2 years and older, or falls of $>3$ feet for those $<2$ years]); 3) loss of consciousness; 4) Glasgow Coma Scale score at presentation; 5) age; and 6) sex.

The 17 variables identified by radiologists on CT scans included the presence or absence of the following: cerebellar hemorrhage, cerebral contusion, cerebral edema, cerebral hemorrhage/intracerebral hematoma, diastasis of the skull, epidural hematoma, extraaxial hematoma, intraventricular hemorrhage, midline shift/shift of brain structures, pneumocephalus, skull fracture (including cerebrospinal fluid leak), subarachnoid hemorrhage, subdural hematoma, traumatic infarction, diffuse axonal injury, herniation, and shear injury. Head CT scans were interpreted by attending radiologists at each clinical site, and a blinded pediatric radiologist made definitive interpretations on scans that were difficult to interpret. ${ }^{19}$ Each site was responsible for ensuring the accuracy of its data reported to PECARN. In addition, a subsequent study detailed consistent interrater reliability of all data collected by the PECARN consortium. ${ }^{22}$

Our outcome of interest was a CRTBI, a composite of several variables as defined by the PECARN investigators. ${ }^{19}$ The CRTBI variables consisted of any of the following: 1) neurosurgical procedure (e.g., dural repair for cerebrospinal fluid leak, fracture elevation, hematoma drainage, intracranial pressure monitor placement, lobectomy, tissue debridement, ventriculostomy, and "other" neurosurgical procedure), 2) intubated $>24$ hours as a direct result of the head trauma, 3) hospitalization $\geq 48$ hours and evidence of TBI on a head CT scan, and 4) death due to TBI.

\section{ANN Analysis}

We trained an ANN using offline MATLAB R2016b (version 9.1.0.441655) on a 64-bit MacBook Pro running OS 10.11.6. We randomly partitioned patients into 3 groups in order to provide holdout validation on our large data set: $70 \%$ were for training the ANN; $15 \%$ were for validating the ANN; and $15 \%$ were for subsequent final testing of the ANN. The ANN had not been exposed to any of the final test patients until after the model was finished training and validating. A two-layer, feed-forward ANN with 11 sigmoid hidden and softmax output neurons was trained using the scaled conjugate gradient back-propagation method on the dedicated partition. We tabulated confusion tables and statistics on the testing partition, as well as for the entire data set. We assessed the predictive ability of the model rigorously with various numerical measures of accuracy, precision, and error.

\section{Results}

In this study, we included 12,902 patients of whom $63 \%$ were male and the average age was $7.99 \pm 5.91$ years (Table 1). Of the 12,902 patients, 480 suffered a CRTBI. Aside 
TABLE 1. Characteristics of pediatric TBI patients studied using an ANN

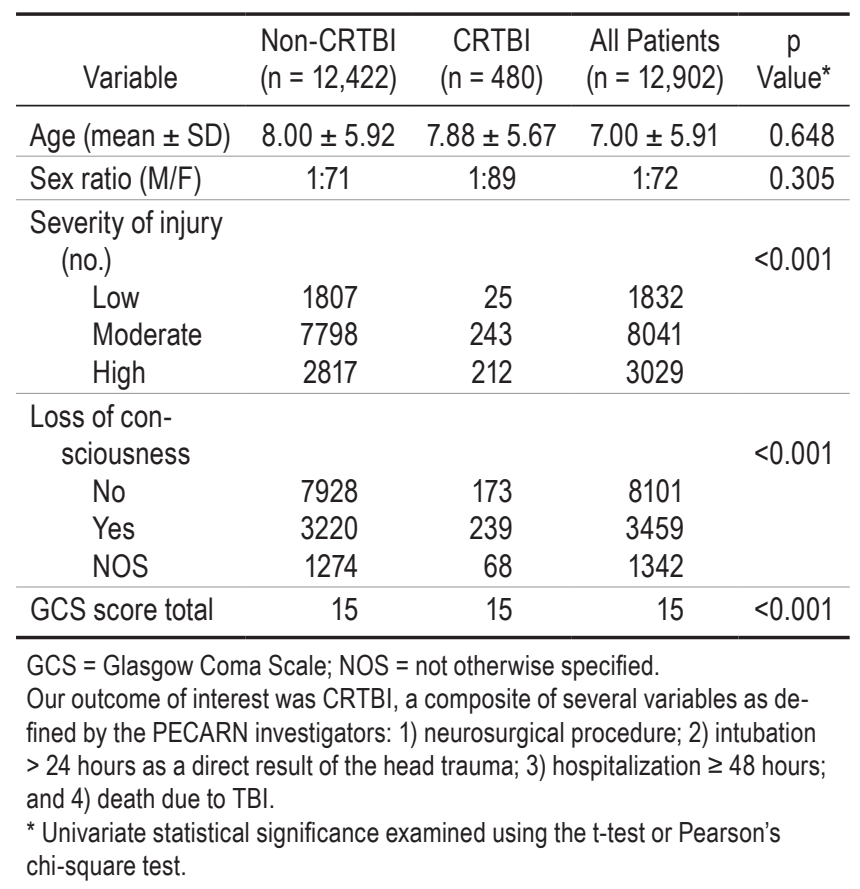

from age and sex, all other clinical and imaging variables had a univariate association with CRTBI (Table 2).

The ANN has a sensitivity of $99.73 \%$ and a negative predictive value (NPV) of $91.23 \%$ for CRTBI in the testing cohort (Table 3). When the data used for testing were combined with the remaining $85 \%$ of data, which the network was trained and validated on, the sensitivity remained very high at $99.54 \%$ and NPV of $84.38 \%$ (Table 3). Determination of specificity was much lower at $60.47 \%$ for testing and $64.17 \%$ for the entire data set. We included other statistical measures of the ANN binary classifier (Table 3 ). A pictorial representation of the ANN constructed here is shown in Fig. 1.

Receiver operator characteristic (ROC) curves for both test patients and the entire data set were calculated (Fig. 2). The area under the ROC curve (AUC) was 0.9907 for prediction of a CRTBI in test patients and 0.9790 for the entire data set.

\section{Discussion}

We constructed and validated an ANN, a machinelearning computational algorithm, to predict CRTBIs in children using clinical and imaging data. This platform has apparent clinical utility for the inexperienced pediatric emergency care provider for assigning admission for children with TBI given its very high sensitivity for CRTBI, which, while it has a low prevalence $(<5 \%)$, can have serious consequences, such as future intracranial procedure(s), respiratory failure, prolonged hospitalization, and/or death. ${ }^{33}$ This would be the first study, to our knowledge, aiming to predict TBI of any type in any patient population.

Predictive outcome and prognostication models are
TABLE 2. Head CT findings of pediatric TBI patients studied using an ANN

\begin{tabular}{|c|c|c|c|}
\hline Imaging Findings & $\begin{array}{l}\text { Non-CRTBI } \\
(n=12,422)\end{array}$ & $\begin{array}{c}\text { CRTBI } \\
(n=480)\end{array}$ & $\begin{array}{l}\text { All Patients } \\
(n=12,902)\end{array}$ \\
\hline \multicolumn{4}{|l|}{ Cerebellar hemorrhage } \\
\hline No & 12418 & 469 & 12887 \\
\hline Yes & 4 & 11 & 15 \\
\hline \multicolumn{4}{|l|}{ Cerebral contusion } \\
\hline No & 12365 & 365 & 12730 \\
\hline Yes & 57 & 115 & 172 \\
\hline \multicolumn{4}{|l|}{ Cerebral edema } \\
\hline No & 12415 & 412 & 12827 \\
\hline Yes & 7 & 68 & 75 \\
\hline \multicolumn{4}{|c|}{$\begin{array}{l}\text { Cerebral hemorrhage or intra- } \\
\text { cerebral hematoma }\end{array}$} \\
\hline No & 12384 & 381 & 12765 \\
\hline Yes & 38 & 99 & 137 \\
\hline \multicolumn{4}{|l|}{ Diastasis of the skull } \\
\hline No & 12403 & 445 & 12848 \\
\hline Yes & 19 & 34 & 54 \\
\hline \multicolumn{4}{|l|}{ Epidural hematoma } \\
\hline No & 12402 & 394 & 12796 \\
\hline Yes & 20 & 86 & 106 \\
\hline \multicolumn{4}{|l|}{ Extraaxial hematoma } \\
\hline No & 12358 & 411 & 12769 \\
\hline Yes & 64 & 69 & 133 \\
\hline \multicolumn{4}{|l|}{ Intraventricular hemorrhage } \\
\hline No & 12415 & 456 & 12871 \\
\hline Yes & 7 & 24 & 31 \\
\hline \multicolumn{4}{|c|}{ Midline shift of brain structures } \\
\hline No & 12416 & 401 & 12817 \\
\hline Yes & 6 & 79 & 85 \\
\hline \multicolumn{4}{|l|}{ Pneumocephalus } \\
\hline No & 12361 & 363 & 12724 \\
\hline Yes & 61 & 117 & 178 \\
\hline \multicolumn{4}{|l|}{ Skull fracture } \\
\hline No & 11828 & 184 & 12012 \\
\hline Yes & 594 & 296 & 890 \\
\hline \multicolumn{4}{|l|}{ Subarachnoid hemorrhage } \\
\hline No & 12356 & 369 & 12725 \\
\hline Yes & 66 & 111 & 177 \\
\hline \multicolumn{4}{|l|}{ Subdural hematoma } \\
\hline No & 12348 & 337 & 12685 \\
\hline Yes & 74 & 143 & 217 \\
\hline \multicolumn{4}{|l|}{ Traumatic infarction } \\
\hline No & 12422 & 476 & 12898 \\
\hline Yes & 0 & 4 & 4 \\
\hline \multicolumn{4}{|l|}{ Diffuse axonal injury } \\
\hline No & 12422 & 475 & 12897 \\
\hline Yes & 0 & 5 & 5 \\
\hline \multicolumn{4}{|l|}{ Herniation } \\
\hline No & 12422 & 475 & 12897 \\
\hline Yes & 0 & 5 & 5 \\
\hline \multicolumn{4}{|l|}{ Shear injury } \\
\hline No & 12419 & 466 & 12885 \\
\hline Yes & 3 & 14 & 17 \\
\hline
\end{tabular}


TABLE 3. Confusion table statistics: testing results of an ANN on pediatric TBI patients

\begin{tabular}{lcc}
\hline \multirow{2}{*}{ Measure } & \multicolumn{2}{c}{ Results } \\
\cline { 2 - 3 } & Test Group Patients & All Patients \\
\hline Sensitivity & 0.9973 & 0.9954 \\
\hline Specificity & 0.6047 & 0.6417 \\
\hline Precision & 0.9819 & 0.9863 \\
\hline NPV & 0.9123 & 0.8438 \\
\hline False-positive rate & 0.3953 & 0.3583 \\
\hline False discovery rate & 0.0181 & 0.0137 \\
\hline Accuracy & 0.0027 & 0.0046 \\
\hline F1 score & 0.9798 & 0.9823 \\
\hline Matthews correlation coefficient & 0.7337 & 0.7272 \\
\hline Area under ROC curve & 0.9907 & 0.9790 \\
\hline
\end{tabular}

We randomly partitioned patients into 3 groups in order to provide holdout validation on our large data set: $70 \%$ were for training the ANN; $15 \%$ were for validating the ANN; and $15 \%$ were for subsequent final testing of the ANN. Various measures of predictive ability on the test patients, as well as test patients combined with those used for training and validation, are presented as proportions.

becoming increasingly important across medicine and surgery (e.g., $\mathrm{CHA}_{2} \mathrm{DS}_{2}$-VASc score, ${ }^{11,12}$ APACHE, ${ }^{17}$ SOFA $^{39}$ ), and modeling techniques have evolved over the years. These models have classically relied on logistic regression or conventional statistics to generate predictions, and often they use fewer input variables that are manually entered. More recently, ANNs have been shown to robustly predict complications, outcomes, and prognosis among numerous fields, ${ }^{5,13,31,34-36}$ including TBI. . $18,26,32,37,38$ Thus, an ANN tool yielding predictive information concerning CRTBI would be helpful and provide an evidence-based mechanism for treating these patients.

ANNs are computational constructs used to interpret the maximum number of combinations of data in complex systems, such as making medical diagnoses in neurosurgery, ${ }^{36,37}$ where many competing factors influence outcome. ${ }^{2}$ During training of the ANN, random "weights" are assigned to each input variable, compared against every variable in the model, and are then used to predict the strength of correlation with the outcome of interest (Fig. 1). While there is no maximum number of variables that can be included in an ANN, addition of irrelevant variables will not make the data prediction any stronger. ${ }^{41}$ Thus, we chose to rationally design the ANN described here by only including variables that had previously been shown by univariate statistical analyses to be significantly associated with CRTBIs.

We trained an ANN on data collected from PECARN and successfully developed a very sensitive (sensitivity 99.73\%, AUC 0.9907) tool for identifying CRTBI (NPV 91\%) in children. We optimized the ANN for sensitivity over specificity to conservatively identify patients likely to be diagnosed with a CRTBI. Future iterations of this ANN with additional variables and data not available through PECARN could be similarly leveraged to optimize specificity, thereby safely ruling out disease. However, since PECARN is a group consisting of 25 hospitals with data collected prospectively, these data most accurately reflect the epidemiological and treatment diversity seen across North America for pediatric TBI. Importantly, the number of variables included in the predictive ANN algorithm can be greatly increased compared with prior risk-calculation tools due to the overwhelmingly computational superiority of machine-learning over conventional statistical approaches, which are limited by degrees of freedom..$^{29,31,37,41}$ Our intent was to provide software that allows for real-world incorporation of data into a standalone application or an electronic medical record (EMR). Future applications could self-collect these clinical data (i.e., our published algorithm depends on collected and interpreted data) and therefore would integrate all necessary input data from EMR systems and provide results for the clinician on the ground. Another strength of this study is its external generalizability, as we did not further divide our cohort based on any further head-injury patterns (e.g., subdural, epidural, intraparenchymal hematoma, shear) often seen in the literature, as we wanted to reflect the full spectrum of pediatric patients with all severities and pathoanatomical types of TBI presenting to any emergency department.

In the our opinion, future iterations of ANN-based predictive modeling should be centered around 3 guiding principles: 1) prospective data collection leading to real-time updates and refinement of the algorithm, 2) direct linkage of ANN models to the EMRs, and 3) increase in the granularity of data available for training the $\mathrm{ANN}$-for instance, using image-based processing. First, compared to traditional statistical approaches that require new analyses to be performed each time new data are added, ANNs can be constantly updated, providing realtime, up-to-date information and quantitative evidence. ANNs could be designed to use national, regional, or even provider-specific data. Second, directly linking ANNs to the EMR would provide streamlined data collection and up-to-date predictive capabilities based on the most current evidence. Lastly, ANNs could be trained directly on the CT images themselves, leading to a quicker diagnosis, prognosis, and better utilization of hospital resources.

Although we lay the groundwork for incorporating machine-learning into evaluation of children with TBI, this study is not without limitations. First, because machine-learning algorithms are computational constructs that are not familiar to most physicians, these models can be seen as foreign and/or unproven entities. ${ }^{6}$ However, as the importance of utilizing "big data" increases, utilizing artificial intelligence and machine learning will inevitably be tools used going forward. ${ }^{6,9,24}$ Second, despite the very large number of total patients, the number of patients in each individual subset of CRTBI was low. However, with additional data, we believe we can create more sophisticated models with higher specificity in the future, providing even better data on who can be safely discharged without risk for readmission. Furthermore, we were not able to incorporate standardized metrics observed during the patient's physical examination, details that are difficult to quantify and capture. Thus, while algorithm-based decision tools can be useful in guiding the physician's decision, these constructs absolutely do not replace the 
Hale et al.

\section{Input Variables}

Cerebellar hemorrhage

Cerebral contusion

Cerebral edema

Cerebral hemorrhage or intracerebral hematoma

Diastasis of the skull

Epidural hematoma

Extra-axial hematoma

Intraventricular hemorrhage

Midline shift

Pneumocephalus

Skull fracture

Subarachnoid

hemorrhage

Subdural hematoma

Traumatic infarction

Diffuse axonal injury

Herniation

Shear injury

\section{Training Nodes}

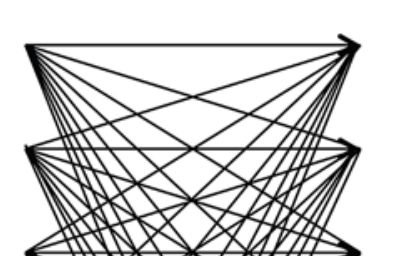

N.

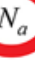

$\mathrm{N}_{0}$

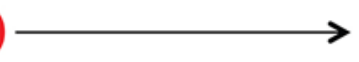

.

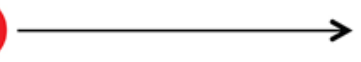

$N_{d}$

(2)

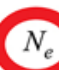

$N_{0}$

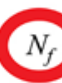

$N_{f}$

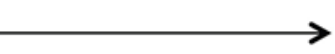

$N_{g}$

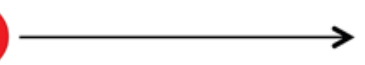

$N_{h}$

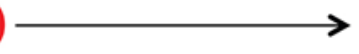

$N_{i}$

$N_{j}$

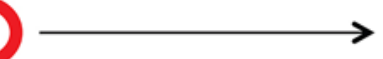

\section{Target Layer}

\section{Outcome: \\ Clinically relevant traumatic brain injury}

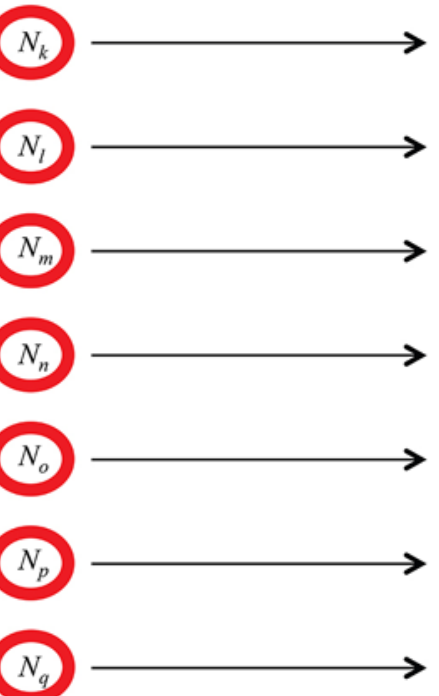

FIG. 1. Schematic of the artificial neural network (ANN) constructed here. Seventeen input variables were compared, converging on more than 100 training nodes (for simplicity, fewer nodes are shown). Each input variable connects, analogous to projections in neurons, to each training node. Arbitrary "weights" are then applied to each variable. Each training node is then used to determine the best "weights" of each variable to predict the outcome of interest ("target layer"). Figure is available in color online only.

information that can only be obtained by a trained physician. Third, each patient in our study underwent head CT scanning, an assumption in itself that our model is heavily dependent on, and the decision to order scans is not standardized across institutions and likely changes over time.
There has been extensive literature presented on the utility and safety of head CT for mild TBI in children since the time these data were collected. ${ }^{23}$ These imaging data have been dichotomized without providing further quantification per covariate (e.g., degree of midline shift, quan- 

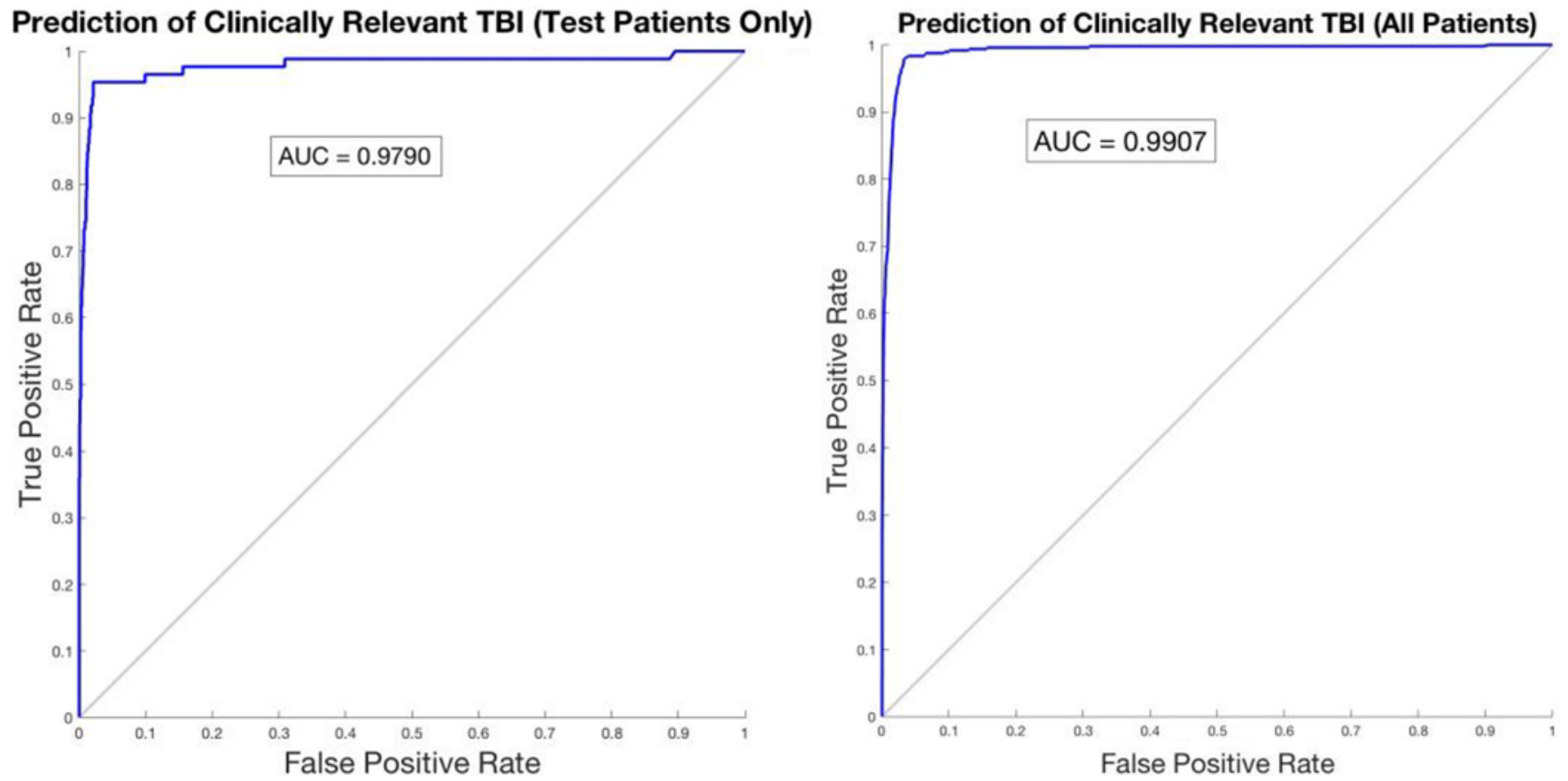

FIG. 2. ROC curve for ANN predictions of CRTBIs. We randomly partitioned patients into 3 groups in order to provide holdout validation on our large data set: $70 \%$ were for training the ANN; $15 \%$ were for validating the ANN; and $15 \%$ were for subsequent final testing of the ANN. The ANN had not been exposed to any of the test patients until after the model was finished training. The ROC for the testing set of patients (left) and the ROC for the entire data set (right). Figure is available in color online only.

tification of hemorrhage). In reality, these CT images are interpreted by a combination of emergency medicine and/ or night-hawk radiologists, such that decisions would be made long before a complex research-level interpretation could be accomplished. Last, we used a single data source (PECARN) that is publicly available and has undergone rigorous quality improvement. However, we are limited by the clinical-practice standards of those years (20042006), including the rationale and threshold to obtain head CT scans in children. Further computational restructuring of our ANN model may also provide additional metrics for future studies that analyze the CT data directly instead of the radiologist's interpretation.

We posit that in-hospital use of the model may actually increase the power of the algorithm as ANNs can be trained on new data, and the model has the potential to be implemented as a future online tool or packaged into the EMR system (available for download in the Supplemental Material), but this would require much of the heavy research-level classification to be performed immediately for this to be time sensitive and clinically relevant. Currently, much of the trauma registry classifications, clinical documentation, and final imaging reads are done well after clinical decisions are made and often times are only fully complete well after patient discharge or death.

\section{Conclusions}

Training an ANN model using data from PECARN, we constructed a highly sensitive tool to diagnose CRTBIs. Further iterations of this ANN may bring real-time, datadriven updates to the hands of pediatric emergency person- nel in order to provide the most accurate evidence-based care and may particularly aid midlevel and/or inexperienced practitioners in small outlying or austere facilities. Immediate identification of pediatric TBI patients who are likely to require additional hospital resources allows clinical teams and hospital administrators to work synergistically to provide the best clinical care. We believe that approaches like our ANN can offer more robust and accurate predictions that can be updated prospectively in real time.

\section{Acknowledgments}

A.T.H. receives salary and tuition support through the Vanderbilt University School of Medicine Medical Scientist Training Program (5T32GM007347). This project is supported by the Vanderbilt Clinical and Translational Science Award (UL1RR024975, NIH/NCAT; to M.B.P.). M.B.P. is funded by R01 (GM120484, NIH/NIGMS) and a Vanderbilt Faculty Scholars Award.

We would also like to thank the Surgical Outcomes Center for Kids at Monroe Carell Jr. Children's Hospital of Vanderbilt University for administrative support.

\section{References}

1. Ahmed FE: Artificial neural networks for diagnosis and survival prediction in colon cancer. Mol Cancer 4:29, 2005

2. Amato F, López A, Peña-Méndez EM, Vaňhara P, Hampl A, Havel J: Artificial neural networks in medical diagnosis. J Appl Biomed 11:47-58, 2013

3. Babl FE, Borland ML, Phillips N, Kochar A, Dalton S, McCaskill M, et al: Accuracy of PECARN, CATCH, and CHALICE head injury decision rules in children: a prospective cohort study. Lancet 389:2393-2402, 2017

4. Badawy MK, Dayan PS, Tunik MG, Nadel FM, Lillis KA, 
Miskin M, et al: Prevalence of brain injuries and recurrence of seizures in children with posttraumatic seizures. Acad Emerg Med 24:595-605, 2017

5. Baxt WG: Application of artificial neural networks to clinical medicine. Lancet 346:1135-1138, 1995

6. Beam AL, Kohane IS: Translating artificial intelligence into clinical care. JAMA 316:2368-2369, 2016

7. Borgialli DA, Mahajan P, Hoyle JD Jr, Powell EC, Nadel FM, Tunik MG, et al: Performance of the pediatric Glasgow Coma Scale score in the evaluation of children with blunt head trauma. Acad Emerg Med 23:878-884, 2016

8. Chong SL, Liu N, Barbier S, Ong ME: Predictive modeling in pediatric traumatic brain injury using machine learning. BMC Med Res Methodol 15:22, 2015

9. Darcy AM, Louie AK, Roberts LW: Machine learning and the profession of medicine. JAMA 315:551-552, 2016

10. Dayan PS, Holmes JF, Hoyle J Jr, Atabaki S, Tunik MG, Lichenstein R, et al: Headache in traumatic brain injuries from blunt head trauma. Pediatrics 135:504-512, 2015

11. Gage BF, van Walraven C, Pearce L, Hart RG, Koudstaal PJ, Boode BS, et al: Selecting patients with atrial fibrillation for anticoagulation: stroke risk stratification in patients taking aspirin. Circulation 110:2287-2292, 2004

12. Gage BF, Waterman AD, Shannon W, Boechler M, Rich MW, Radford MJ: Validation of clinical classification schemes for predicting stroke: results from the National Registry of Atrial Fibrillation. JAMA 285:2864-2870, 2001

13. Gholipour C, Rahim F, Fakhree A, Ziapour B: Using an artificial neural networks (ANNs) model for prediction of intensive care unit (ICU) outcome and length of stay at hospital in traumatic patients. J Clin Diagn Res 9:OC19-OC23, 2015

14. Glass T, Ruddy RM, Alpern ER, Gorelick M, Callahan J, Lee L, et al: Traumatic brain injuries and computed tomography use in pediatric sports participants. Am J Emerg Med 33:1458-1464, 2015

15. Greenberg JK, Yan Y, Carpenter CR, Lumba-Brown A, Keller MS, Pineda JA, et al: Development and internal validation of a clinical risk score for treating children with mild head trauma and intracranial injury. JAMA Pediatr 171:342-349, 2017

16. Ide K, Uematsu S, Tetsuhara K, Yoshimura S, Kato T, Kobayashi T: External validation of the PECARN head trauma prediction rules in Japan. Acad Emerg Med 24:308-314, 2017

17. Knaus WA, Draper EA, Wagner DP, Zimmerman JE: APACHE II: a severity of disease classification system. Crit Care Med 13:818-829, 1985

18. Kreif N, Grieve R, Díaz I, Harrison D: Evaluation of the effect of a continuous treatment: a machine learning approach with an application to treatment for traumatic brain injury. Health Econ 24:1213-1228, 2015

19. Kuppermann N, Holmes JF, Dayan PS, Hoyle JD Jr, Atabaki SM, Holubkov R, et al: Identification of children at very low risk of clinically-important brain injuries after head trauma: a prospective cohort study. Lancet 374:1160-1170, 2009

20. Lee LK, Monroe D, Bachman MC, Glass TF, Mahajan PV, Cooper A, et al: Isolated loss of consciousness in children with minor blunt head trauma. JAMA Pediatr 168:837-843, 2014

21. Magana JN, Kuppermann N: The PECARN TBI rules do not apply to abusive head trauma. Acad Emerg Med 24:382384, 2017

22. Marcin JP, Romano PS, Dharmar M, Chamberlain JM, Dudley N, Macias CG, et al: Implicit review instrument to evaluate quality of care delivered by physicians to children in emergency departments. Health Serv Res 53:1316-1334, 2018

23. Miglioretti DL, Johnson E, Williams A, Greenlee RT, Weinmann S, Solberg LI, et al: The use of computed tomography in pediatrics and the associated radiation exposure and estimated cancer risk. JAMA Pediatr 167:700-707, 2013

24. Murdoch TB, Detsky AS: The inevitable application of big data to health care. JAMA 309:1351-1352, 2013

25. Nakhjavan-Shahraki B, Yousefifard M, Hajighanbari MJ, Oraii A, Safari S, Hosseini M: Pediatric Emergency Care Applied Research Network (PECARN) prediction rules in identifying high risk children with mild traumatic brain injury. Eur J Trauma Emerg Surg 43:755-762, 2017

26. Nielson JL, Cooper SR, Yue JK, Sorani MD, Inoue T, Yuh EL, et al: Uncovering precision phenotype-biomarker associations in traumatic brain injury using topological data analysis. PLoS One 12:e0169490, 2017

27. Nigrovic LE, Lee LK, Hoyle J, Stanley RM, Gorelick MH, Miskin M, et al: Prevalence of clinically important traumatic brain injuries in children with minor blunt head trauma and isolated severe injury mechanisms. Arch Pediatr Adolesc Med 166:356-361, 2012

28. Nigrovic LE, Lillis K, Atabaki SM, Dayan PS, Hoyle J, Tunik $\mathrm{MG}$, et al: The prevalence of traumatic brain injuries after minor blunt head trauma in children with ventricular shunts. Ann Emerg Med 61:389-393, 2013

29. Obermeyer Z, Emanuel EJ: Predicting the future-big data, machine learning, and clinical medicine. $\mathbf{N}$ Engl J Med 375:1216-1219, 2016

30. Palchak MJ, Holmes JF, Vance CW, Gelber RE, Schauer BA, Harrison MJ, et al: Does an isolated history of loss of consciousness or amnesia predict brain injuries in children after blunt head trauma? Pediatrics 113:e507-e513, 2004

31. Penny W, Frost D: Neural networks in clinical medicine. Med Decis Making 16:386-398, 1996

32. Pourahmad S, Hafizi-Rastani I, Khalili H, Paydar S: Identifying important attributes for prognostic prediction in traumatic brain injury patients. A hybrid method of decision tree and neural network. Methods Inf Med 55:440-449, 2016

33. Quayle KS, Powell EC, Mahajan P, Hoyle JD Jr, Nadel FM, Badawy MK, et al: Epidemiology of blunt head trauma in children in U.S. emergency departments. N Engl J Med 371:1945-1947, 2014

34. Rughani AI, Dumont TM, Lu Z, Bongard J, Horgan MA, Penar PL, et al: Use of an artificial neural network to predict head injury outcome. J Neurosurg 113:585-590, 2010

35. Segal ME, Goodman PH, Goldstein R, Hauck W, Whyte J, Graham JW, et al: The accuracy of artificial neural networks in predicting long-term outcome after traumatic brain injury. J Head Trauma Rehabil 21:298-314, 2006

36. Senders JT, Arnaout O, Karhade AV, Dasenbrock HH, Gormley WB, Broekman ML, et al: Natural and artificial intelligence in neurosurgery: a systematic review. Neurosurgery 83:181-192, 2018

37. Senders JT, Staples PC, Karhade AV, Zaki MM, Gormley WB, Broekman MLD, et al: Machine learning and neurosurgical outcome prediction: a systematic review. World Neurosurg 109:476-486, 486.e1, 2018

38. Senders JT, Zaki MM, Karhade AV, Chang B, Gormley WB, Broekman ML, et al: An introduction and overview of machine learning in neurosurgical care. Acta Neurochir (Wien) 160:29-38, 2018

39. Vincent JL, Moreno R, Takala J, Willatts S, De Mendonça A, Bruining H, et al: The SOFA (Sepsis-related Organ Failure Assessment) score to describe organ dysfunction/failure. On behalf of the Working Group on Sepsis-Related Problems of the European Society of Intensive Care Medicine. Intensive Care Med 22:707-710, 1996

40. Walczak S: Artificial neural network medical decision support tool: predicting transfusion requirements of ER patients. IEEE Trans Inf Technol Biomed 9:468-474, 2005

41. Zou J, Han Y, So SS: Overview of artificial neural networks. Methods Mol Biol 458:15-23, 2008 


\section{Disclosures}

The authors report no conflict of interest concerning the materials or methods used in this study or the findings specified in this paper.

\section{Author Contributions}

Conception and design: Hale, Stonko. Acquisition of data: Hale, Stonko, Lim. Analysis and interpretation of data: Hale, Stonko, Lim, Patel. Drafting the article: Hale. Critically revising the article: all authors. Reviewed submitted version of manuscript: all authors. Approved the final version of the manuscript on behalf of all authors: Hale. Statistical analysis: Hale, Stonko. Study supervision: Shannon, Patel.

\section{Supplemental Information \\ Online-Only Content}

Supplemental material is available with the online version of the article.

Supplemental Material. https://thejns.org/doi/suppl/10.3171/ 2018.8.PEDS18370

\section{Correspondence}

Andrew T. Hale: Vanderbilt University School of Medicine, Nashville, TN. andrew.hale@vanderbilt.edu. 